PROCEEDINGS OF THE

AMERICAN MATHEMATICAL SOCIETY

Volume 93, Number 2, February 1985

\title{
RANDOM POLYTOPES ON THE TORUS
}

\author{
C. BUCHTA AND R. F. TICHY
}

ABSTRACT. The expected volume of the convex hull of $n$ random points chosen independently and uniformly on the $d$-dimensional torus is determined.

In the 1860's J. J. Sylvester raised the problem of determining the expected area $V_{3}(C)$ of the convex hull of three points chosen independently and uniformly at random from a given plane convex body $C$ of area one. For some special plane convex bodies the problem was solved by Woolhouse [16], Crofton [8] and Deltheil $[9]$, e.g. $V_{3}($ triangle $)=1 / 12, V_{3}($ parallelogram $)=11 / 144, V_{3}($ regular hexagon $)=$ $289 / 3888, V_{3}($ ellipse $)=35 / 48 \pi^{2}$. More generally, Alikoski $[\mathbf{1}]$ proved that

$$
V_{3}(\text { regular } m \text {-gon })=\frac{9 \cos ^{2} \omega+52 \cos \omega+44}{36 m^{2} \sin ^{2} \omega} \quad\left(\omega=\frac{2 \pi}{m}\right) .
$$

For any convex polygon $C$ and an arbitrary number $n$ of random points, the expected area $V_{n}(C)$ of their convex hull was determined in a recent paper [5]. For example,

$$
\begin{gathered}
V_{n}(\text { triangle })=1-\frac{2}{n+1} \sum_{k=1}^{n} \frac{1}{k}, \\
V_{n}(\text { parallelogram })=1-\frac{8}{3(n+1)}\left[\sum_{k=1}^{n+1} \frac{1}{k}\left(1-\frac{1}{2^{k}}\right)-\frac{1}{(n+1) 2^{n+1}}\right] .
\end{gathered}
$$

In 1917 Blaschke $[3,4]$ succeeded in proving that, among all plane convex bodies $C$ of area one, $V_{3}(C)$ attains its minimum if $C$ is an ellipse. In 1974 Groemer [11, 12] generalized this statement to $d$-dimensional convex bodies $C$ of volume one and an arbitrary number $n$ of points. The value of this minimum is known for $d+1$ points in a $d$-dimensional ellipsoid (Kingman [13]) and for an arbitrary number of points in dimensions $d=2$ and $d=3$ (cf. [6]).

An obvious question is to ask for the expected volume of the convex hull of random points chosen on a compact metric manifold. A subset $C$ of a compact metric manifold is called convex (cf. Bangert [2], Walter [14]) if for any two points $x, y \in C$ all geodesic segments (i.e. all curves of minimal length on the manifold joining $x$ and $y$ ) are completely contained in $C$. The convex hull of a set is the smallest convex set (on the manifold) containing it.

In the case of the $d$-dimensional sphere $S^{(d)}=\left\{x \in E^{d+1}:\|x\|=1\right\}\left(E^{d+1}\right.$ denotes $(d+1)$-dimensional Euclidean space), the metric is defined by the minimal Euclidean length of all curves on $S^{(d)}$ joining two points. A convex set on $S^{(d)}$ is either contained in a hemisphere or is the sphere itself. If $n$ points are contained

Received by the editors January 17, 1984 and, in revised form, March 26, 1984 .

1980 Mathematics Subject Classification. Primary 52A22; Secondary 60D05. 
in some common hemisphere, their convex hull has the same property with probability one. As shown by Wendel [15], the probability $p_{n}^{(d)}$ that $n$ points chosen independently and uniformly at random from (the normalized measure space) $S^{(d)}$ lie on some common hemisphere is given by

$$
p_{n}^{(d)}=\frac{1}{2^{n-1}} \sum_{k=0}^{d}\left(\begin{array}{c}
n-1 \\
k
\end{array}\right)
$$

Further, Cover and Efron [7] showed for the expected volume $\bar{V}_{n}^{(d)}$ of the convex hull of $n$ such points, on condition that all points lie on some common hemisphere, that

$$
\bar{V}_{n}^{(d)}=\left(\begin{array}{c}
n-1 \\
d
\end{array}\right) /\left(2 \sum_{k=0}^{d}\left(\begin{array}{c}
n-1 \\
k
\end{array}\right)\right)=\left(\begin{array}{c}
n-1 \\
d
\end{array}\right) /\left(2^{n} p_{n}^{(d)}\right) .
$$

Formulae (4) and (5) yield the expected volume $V_{n}^{(d)}$ of the convex hull of $n$ points chosen independently and uniformly on $S^{(d)}$ :

$$
V_{n}^{(d)}=p_{n}^{(d)} \bar{V}_{n}^{(d)}+\left(1-p_{n}^{(d)}\right) \cdot 1=1-p_{n}^{(d)}\left(1-\bar{V}_{n}^{(d)}\right) .
$$

In contrast to the sphere, the torus has apparently not been investigated.

The $d$-dimensional torus $T^{(d)}$ is the quotient space $E^{d} / Z^{d}, Z^{d}$ being the system of all lattice points in Euclidean $d$-space $E^{d}$. By

$$
\rho(x, y)=\inf _{l \in Z^{d}}\|x-y+l\|
$$

a metric is defined on $T^{(d)} \cdot\left(T^{(d)}, \mu_{d}\right)$, where $\mu_{d}$ denotes $d$-dimensional Lebesgue measure, is isomorphic to the $d$-fold product of $\left(T^{(1)}, \mu\right), \mu$ being Lebesgue measure on the unit interval:

$$
\left(T^{(d)}, \mu_{d}\right) \cong \bigotimes_{k=1}^{d}\left(T^{(1)}, \mu\right)
$$

$I \subset T^{(1)}$ is called a one-dimensional cell if $I$ is a subinterval of $[0,1)$ or the complement of a subinterval of $[0,1)$. A d-dimensional cell is the Cartesian product of $d$ one-dimensional cells. For a given convex subset $C \subset T^{(d)}$ there exists a smallest cell $C(C)$ containing $C$. It is a Cartesian product of $d$ one-dimensional cells, each of them of length $l<1 / 2$ or $l=1$.

Let $t=t(C)$ denote the number of such one-dimensional cells of length $l=1 ; t$ is called the type of $C$. Note that $t$ is an integer, $0 \leq t \leq d$ and $t\left(T^{(d)}\right)=d$.

THEOREM. Denote by $p_{n}^{(d)}(t)$ the probability that the convex hull $H_{n}$ of $n$ points chosen independently and uniformly at random on the d-dimensional torus $T^{(d)}$ is of type $t$, by $V_{n}^{(d)}(t)$ the expected volume of $H_{n}$ on condition that $H_{n}$ is of type $t$, and by $W_{n}^{(d)}$ the expected volume of the convex hull of $n$ points chosen independently and uniformly at random from the d-dimensional Euclidean unit cube; $W_{n}^{(0)}:=1$. Then

$$
p_{n}^{(d)}(t)=\left(\begin{array}{l}
d \\
t
\end{array}\right)\left(\frac{n}{2^{n-1}}\right)^{d-t}\left(1-\frac{n}{2^{n-1}}\right)^{t}
$$




$$
V_{n}^{(d)}(t)=\left(\frac{n+1}{2 n}\right)^{d-t} W_{n}^{(d-t)}
$$

Thus, the expected volume $V_{n}^{(d)}$ of the convex hull of $n$ points chosen independently and uniformly at random on $T^{(d)}$ is given by

$$
V_{n}^{(d)}=\sum_{t=0}^{d} p_{n}^{(d)}(t) V_{n}^{(d)}(t)=\sum_{t=0}^{d}\left(\begin{array}{l}
d \\
t
\end{array}\right)\left(\frac{n+1}{2^{n}}\right)^{d-t}\left(1-\frac{n}{2^{n-1}}\right)^{t} W_{n}^{(d-t)}
$$

PROOF. To derive (7) we calculate the probability that the convex hull of $n$ points chosen independently and uniformly from $[0,1)$ is a cell of length $l<1 / 2$. The probability that one of the chosen points is an extreme point of such a cell obviously is $1 / 2^{n-2}$. As all points are independently and uniformly distributed, the expected number of such extreme points is $n / 2^{n-2}$. On the other hand, the expected number of extreme points on condition that all points lie in some common cell of length $l<1 / 2$ is, of course, two. Thus, with probability $n / 2^{n-1}$, the convex hull of $n$ random points in the unit interval is a cell of length $l<1 / 2$, whence (7) follows immediately.

To prove (8) assume that $H_{n}$ is of type $t$. Then the smallest cell $C\left(H_{n}\right)$ containing $H_{n}$ is the Cartesian product of $t$ unit intervals and $d-t$ cells of length $l<1 / 2$. Hence, the expected volume of $C\left(H_{n}\right)$ is $\left(V_{n}^{(1)}(0)\right)^{d-t}$. By (7) the probability that $n+1$ random points chosen from the unit interval lie in some common cell of length $l<1 / 2$ on condition that $n$ points do so is $(n+1) / 2 n$. Therefore, on condition that all points lie in some common cell of length $l<1 / 2$, the probability that one of the $n+1$ random points is an extreme point of the convex hull of these points is given by

$$
\frac{(n+1) / 2 n-V_{n}^{(1)}(0)}{(n+1) / 2 n} .
$$

As the $n+1$ points are independently and uniformly distributed, it follows that the expected number of extreme points of their convex hull on condition that they are contained in some common cell of length $l<1 / 2$ is represented by

$$
(n+1)\left(1-\frac{2 n}{n+1} V_{n}^{(1)}(0)\right) \text {. }
$$

Obviously, the expected number of such extreme points is two, whence

$$
V_{n}^{(1)}(0)=\frac{r-}{2 n} \text {. }
$$

Since the expected length of the convex hull of $n$ random points in a unit interval is $(n-1) /(n+1)$, the ratio of the expected volume of $H_{n}$ to the expected volume of the cell $\mathrm{C}\left(H_{n}\right)$ is given by

$$
\left(\frac{n+1}{n-1}\right)^{d-t} W_{n}^{(d-t)}
$$

Thus,

$$
V_{n}^{(d)}(t)=\left(\frac{n-1}{2 n}\right)^{d-t}\left(\frac{n+1}{n-1}\right)^{d-t} W_{n}^{(d-t)}=\left(\frac{n+1}{2 n}\right)^{d-t} W_{n}^{(d-t)} .
$$

This finishes the proof of the theorem. 
REMARK 1. For $d=1$ the above-mentioned results by Wendel and by Cover and Efron for the sphere are obtained. Our proof is different from the arguments used by these authors.

REMARK 2. In the case $d=2$, formula (3) gives explicit values for $W_{n}^{(2)}=$ $V_{n}$ (parallelogram), further, $W_{n}^{(1)}=(n-1) /(n+1)$ and $W_{n}^{(0)}=1$. Correspondingly,

$$
\begin{gathered}
p_{n}^{(2)}(0)=\left(\frac{n}{2^{n-1}}\right)^{2} \\
V_{n}^{(2)}(0)=\left(\frac{n+1}{2 n}\right)^{2}\left\{1-\frac{8}{3(n+1)}\left[\sum_{k=1}^{n+1} \frac{1}{k}\left(1-\frac{1}{2^{k}}\right)-\frac{1}{(n+1) 2^{n+1}}\right]\right\} \\
p_{n}^{(2)}(1)=\frac{n}{2^{n-2}}\left(1-\frac{n}{2^{n-1}}\right), \quad V_{n}^{(2)}(1)=\frac{n-1}{2 n} \\
p_{n}^{(2)}(2)=\left(1-\frac{n}{2^{n-1}}\right)^{2}, \quad V_{n}^{(2)}(2)=1 .
\end{gathered}
$$

REMARK 3. In order to obtain explicit values in the three-dimensional case, it is necessary to determine $W_{n}^{(3)}$. Considerations similar to investigations by Efron [10] lead to the following integral representation in spherical coordinates: Denote by $p(\phi, \theta)$ the support function of the unit cube $C=[-1 / 2,1 / 2]^{3}$, by $\tilde{V}=\tilde{V}(p, \phi, \theta)$ the volume of the smaller of the two parts of $C$ cut off by the plane $\varepsilon=\varepsilon(p, \phi, \theta)$ orthogonal to the direction $(\phi, \theta)$ and with distance $p$ from the origin, by $\tilde{S}=$ $\tilde{S}(p, \phi, \theta)$ the area of $\varepsilon \cap C$, and by $\tilde{T}=\tilde{T}(p, \phi, \theta)$ the expected area of the convex hull of three random points in $\varepsilon \cap C$. Then

$W_{n}^{(3)}=1-\frac{2}{n+1}-\frac{(n-1) n}{6} \int_{0}^{p(\phi, \theta)} \int_{0}^{2 \pi} \int_{0}^{\pi}\left[\tilde{V}^{n-2}+(1-\tilde{V})^{n-2}\right] \tilde{S}^{3} \tilde{T} \sin \theta d \theta d \phi d p$.

Thus, $\tilde{T}$ has to be determined in order to calculate the integral. The intersection $\varepsilon \cap C$ is a polygon (more precisely a triangle, a parallelogram, a pentagon or a hexagon), and the expected area of a random triangle contained in polygon is derived in [5].

ACKnowledgement. We are obliged to Professor R. Schneider (Freiburg) for references [1, $\mathbf{7}$ and 15] and to Professor R. Schnabl (Wien) for valuable discussions.

\section{REFERENCES}

1. H. A. Alikoski, Über das Sylvestersche Vierpunktproblem, Ann. Acad. Sci. Fenn. Ser. A 51 (1939), $1-10$.

2. V. Bangert, Konvexe Mengen in Riemannschen Mannigfaltigkeiten, Math. Z. 162 (1978), 263-286.

3. W. Blaschke, Lösung des "Vierpunktproblems" von Sylvester aus der Theorie der geometrischen Wahrscheinlichkeiten, Leipziger Berichte 69 (1917), 436-453.

4. __ Vorlesungen über Differentialgeometrie II, Affine Differentialgeometrie, Springer, Berlin, 1923.

5. C. Buchta, Zufallspolygone in konvexen Vielecken, J. Reine Angew. Math. 347 (1984), 212-220.

6. __ Das Volumen von Zufallspolyedern im Ellipsoid, Anz. Österreich. Akad. Wiss. Math.-Natur. Kl., 1984.

7. T. M. Cover and B. Efron, Geometrical probability and random points on a hypersphere, Ann. Math. Statist. 38 (1967), 213-220.

8. M. W. Crofton, Probability, Encyclopaedia Britannica, vol. 19, 1885, pp. 768-788.

9. R. Deltheil, Probabilités géométriques, Traité du Calcul des Probabilités et de ses Applications, Gauthier-Villars, Paris, 1926. 
10. B. Efron, The convex hull of a random set of points, Biometrika 52 (1965), 331-343.

11. H. Groemer, On some mean values associated with a randomly selected simplex in a convex set, Pacific J. Math. 45 (1973), 525-533.

12., On the mean value of the volume of a random polytope in a convex set, Arch. Math. 25 (1974), 86-90.

13. J. F. C. Kingman, Random secants of a convex body, J. Appl. Probab. 6 (1969), 660-672.

14. R. Walter, Konvexität in riemannschen Mannigfaltigkeiten, Jahresber. Deutsch. Math.-Verein. 83 (1981), 1-31.

15. J. G. Wendel, A problem in geometric probability, Math. Scand. 11 (1962), 109-111.

16. W. Woolhouse, Educational Times, 1867.

Institut FUR ANALYSis, TECHNisChe MATHEMATIK UND VersicherungsmatheMAtik, Technische Universität Wien, Gusshausstrasse 27-29, A-1040, Wien, AUSTRIA 